\title{
RESEARCH OPPORTUNITY IDENTIFICATION: A PEDAGOGICAL FRAMEWORK FOR DESIGN RESEARCH
}

\author{
Gaurav VAIDYA and Pratul Ch KALITA \\ Indian Institute of Technology Guwahati, India
}

\begin{abstract}
"Where do I start from?" More often than not, design researchers face this question early on in their research projects. The initial ambiguity frequently results in jumping quickly into the project, which leads to a shift in research focus time and again. To set the initial direction, a researcher needs to have an open mind-set and explore the latest changes occurring in the present world, always thinking about innovative opportunities. Finding new/innovative opportunity areas for carrying out research and defining the initial boundaries of a research space are some of the foremost priorities of a researcher. Although the approach followed by design students is more intuitive and opportunistic to solve any problem, the method followed by other disciplines such as STEM is usually more organized. This often leads to tension when a design research project demands the collaborative work of an interdisciplinary team. In this paper, we share an outcome of a study involving an interdisciplinary team working on identifying an opportunity area for design research. The current investigation is used as a case study to illustrate the key phases and their attributes involved in the framework to identify a significant area for conducting a design research project. The framework developed is found to be handy in organizing our thoughts and setting our initial research intent. Based on the study, we propose a pedagogical framework to better equip design researchers to work in collaboration for recognizing the field and scope of a design research project.
\end{abstract}

Keywords: Research intent, collaborative work, research gap, design research, design education

\section{INTRODUCTION}

A research gap can be defined as a topic or area for which insufficient knowledge restricts researchers' capacity to arrive at a conclusion for a given question [1]. Identifying a research gap or area for investigation often turns out to be difficult for researchers and academicians [2] not because of the limited number of issues available for study but due to the complexity involved in formulating a relevant research gap, which is unique and researchable. For designers, the task becomes more challenging due to a defining factor called as 'design attitude'. Design attitude is working in a manner that is more 'assertion-based rather than evidence-based' [3]. Furthermore, for a researcher, 'scientific attitude' encourages working, which is more evidence-based supported by conclusive facts and observations [4]. Design attitude and Scientific attitude appear to be contrasting approaches. An interdisciplinary team's operations could become challenging when members of the team function with such conflicting approaches and working styles. In this regard, not much focus is evident in design education. Thus, it is essential to develop a systematic approach in identifying research gap or area of an investigation while functioning as a team consisting of members from different educational backgrounds. This paper aims to explore and propose a new pedagogical framework to address this issue through investigations where an interdisciplinary team is working to identify opportunity area or research gap for design research. The proposed framework (as shown in figure 3) consists of four stages explore, categorize, summarize, and evaluate. At each stage, specific team members take the lead to steer the process.

\section{FORMATIONS OF AN INTERDISCIPLINARY TEAM AND TEAM ROLES}

For the current study, an interdisciplinary team originated at the Indian Institute of Technology Guwahati in Assam, India, to identify an opportunity area for conducting design research. The team consisted of a diverse mix of members from Product Design, Interaction Design, Human Factors, 
Engineering, and Architecture. According to Belbin, any team has two parts, a functional role, and a team role. The functional role is nothing but the specialized skills a member possesses to perform a profession-based task, and the team role is the attitude and behaviour of a member that is put to use for the effective working of a team [5]. Further, Belbin stated nine team roles that can be grouped in three broad categories: action-oriented roles - Shaper, Implementer and Completer Finisher; people-oriented roles - Co-ordinator, Team worker, and Resource Investigator; cerebral roles - Plant, Monitor Evaluator and Specialist [6]. These team roles at the different stages of the proposed framework are as shown in figure 1.

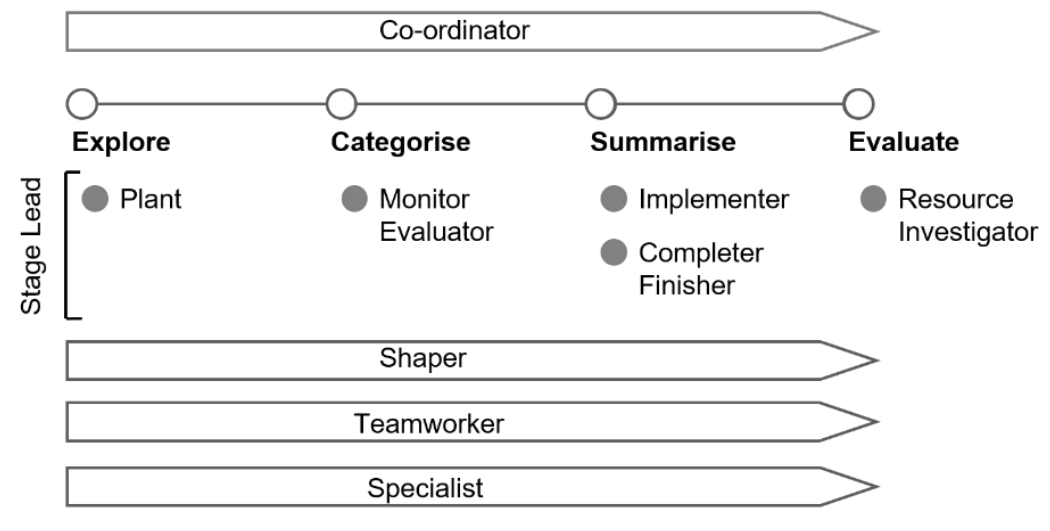

Figure 1. Team Roles

In the interdisciplinary team formed, the team roles are well-defined, and the necessary members are involved at the required time by the Co-ordinator. The role of a Co-ordinator is to oversee the entire process and assign appropriate people to lead at different stages of the process [7]. Only required people at each stage will mean less frustration (and fewer time-consuming meetings) for everyone involved. Designers often play the role of a Plant in a team. Plants are effective in tackling complex early stages of a process innovatively through their creative thinking skills. The Shaper keeps the project stick to the deadlines. Teamwork is essential for empathy and averting friction, and the Specialist is necessary when in-depth understanding is called for. The Monitor Evaluator team role, which is to see all the options, strategize and judge accurately [5], would be best suited for the Categories stage to lead the process. Implementer and Completer Finisher can be considered to lead for the Summaries stage because of the qualities like the ability to turn ideas into practical action-based tasks and deliver on time. The Resource Investigator is assigned as a lead in the Evaluation stage, which requires the development of contacts and communication with people through interviews and surveys.

\section{THE PROCESS OF RESEARCH OPPORTUNITY IDENTIFICATION}

To set the initial direction, researchers need to examine various areas, and it requires a lot of reading and analysing the material from multiple resources. This initial exploration follows a repetitive loop of search, communicate, and group think. As Tom suggested, "First, you need to identify and select relevant information sources, which will mean looking at books in the library, catalogues, databases and on the Internet" [8]. The Plant leads the Explore stage.

\subsection{Explore}

Search - Researchers need to dedicate specific time to search present-day information from various sources keeping an open mind for all kinds of latest information without any bias. An unbiased outlook, in the beginning, can help to find new directions.

Communicate - The information collected gathered from the sources should be compiled. The compiled information can have a format that can be easily understood and quickly shared with others to add comments and highlight critical points while going through it (figure 2).

Group Think - Team discussions with a set meeting agenda and specific time limit allow the ideas to exchange through sharing thoughts. It will create understandings of how information is gathered and steer the project in a definite direction. The team can go back to search for information about the trends that emerged during group think. 


\begin{tabular}{|c|c|c|c|}
\hline Area & Keywords & Key Points & Source \\
\hline Transportation & $\begin{array}{l}\text { Electric Vehicles, Self-drive, Air } \\
\text { Pollution, Batteries, Clean Energy, } \\
\text { Safety }\end{array}$ & $\begin{array}{l}\text { 1. The spread of EVs may open up opportunities to provide } \\
\text { storage support for renewables via the so-called "vehicle-to- } \\
\text { grid" (V2G) option. } \\
\text { 2. There could be significant advantages from using vehicle-to- } \\
\text { grid and associated home-based smart power and storage } \\
\text { systems. } \\
\text { 3. Whether autonomous self-driving car takes off remains to be } \\
\text { seen. Safety is a key issue, with some high-profile and fatal } \\
\text { accidents occurring with early versions. }\end{array}$ & $\begin{array}{l}\text { PhysicsWorld (https:// } \\
\text { physicsworld.com/a/an- } \\
\text { electric-car-future/) } \\
\text { Accessed on: 20-11-2019 }\end{array}$ \\
\hline Healthcare & $\begin{array}{l}\text { Artificial intelligence, Virtual } \\
\text { Reality, Augmented Reality, } \\
\text { Trackers, Sensors }\end{array}$ & $\begin{array}{l}\text { 1. Google's DeepMind created an A.I. for breast cancer analysis. } \\
\text { The alporithm outperformed all human radiologists on pre- } \\
\text { selected data sets to identify breast cancer, on average by } 11.5 \% \text {. } \\
\text { 2. VR is being used to train future surgeons and for actual } \\
\text { surgeons to practice operations. } \\
\text { 3. In case of medical professionals, AR might help medical } \\
\text { students prepare better for real-life operations, as well as } \\
\text { enables surgeons to enhance their capabilities. }\end{array}$ & $\begin{array}{l}\text { The medical futurist (https:/ } \\
\text { medicalfuturist.com/ten- } \\
\text { ways-technology-changing- } \\
\text { healthcare/) Accessed on: }\end{array}$ \\
\hline
\end{tabular}

Figure 2. Sample compiled information with critical points highlighted

\subsection{Categorize}

The initial efforts to explore the latest information would generate an extensive database of current issues (raw data). This information needs to be organized into a limited number of relevant areas. These areas can emerge through team discussions. Articulation of relevance of these areas and type of information each area would contain helps to manage time and keeps focus on the suitable context of the research. Moreover, an additional search could be conducted in the focus area. In the current study, five areas emerged: technology, society, culture, policy, and business. The relevant area of research was decided to be related to 'smart wearable technology' through team discussions. Further, to dig deeper into the area, several queries are raised about constraints, goals, and desires of the category. For example, what are the technology constraints? what are the technology goals? what are the aspired applications of the technology?

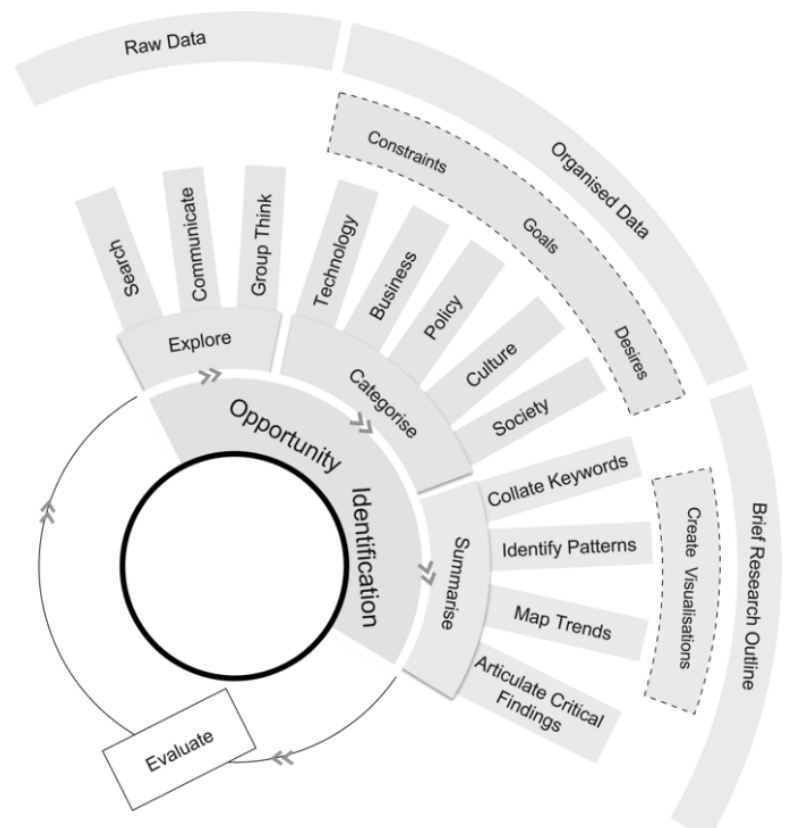

Figure 3. Research Opportunity Identification Process Workflow

\subsection{Summarize}

At the end of the analysis of the latest information gathered in Explore and Categorize modes, we put together the keywords, critical observations, emerging patterns into an integrated summary of research relevant areas. It presents a concise overview of the context and reveals hidden details, trends, insights. It also explains the basic logic behind the research direction. Summarizing the findings is done by collating keywords, identifying patterns, mapping trends, and articulating critical findings.

Collate Keywords - Keywords are nothing but context-specific principal terms that can be used for further search. The use of keywords made the search easier and focused on obtaining relevant information through an extensive database. Analysis of keywords can uncover interesting insights from multiple perspectives. The current investigation identified a total of 1103 relevant research studies to collate keywords. These studies are shortlisted based on the following inclusion criteria: published in 
English from the year 2005 to 2019, indexed in Scopus, screening keywords in their title/abstract/keywords section. The screening keywords used are 'Smart Wearable Devices' and 'Design'. How the identified research documents are distributed is as follows: journal articles (432), conference papers (598), reviews (69), books (4). The identified studies contained a total of 9553 author keywords and index keywords.

Identify Patterns - Identification of the patterns within a dataset is essential to understand the most relevant and evolving areas for the research. One way to find patterns is to cluster the keywords based on their number of occurrences in the articles. Visually representing these patterns becomes an effective way of drawing conclusions and communicating the findings. A total of 171 keywords with a minimum frequency of 15 are selected out of total keywords to generate a network visualization using VOS viewer. The generated network visualization is analysed to discover patterns within the keyword's dataset.

Analysis of the network visualization shows that the selected keywords predominantly form three clusters (represented in red, blue, and green colours). In a cluster, the size of a node (circle) and its label scale represents the weight of an item. Central nodes of these three clusters are identified as 'wearable technology design' (red), 'textiles' (blue), and 'human (physiology monitoring)' (green). The distance between any two nodes represents their relatedness in terms of their co-occurrence in the literature. In other words, the closer the nodes more is their relatedness in terms of co-occurrence. From the network visualization, it appears that the nodes 'product design', 'human-computer interaction', 'human engineering', 'watches' are closely related to the 'wearable technology design' node. Based on this, it is appropriate to believe that product design, human-computer interaction, ergonomics, etc., are the central issues in wearable technology design. From the network visualization, it also appears that the importance of the other two clusters, 'textile' and 'human (physiology monitoring)', is also noteworthy. The 'textile' cluster is more related to smart textiles, use of innovative materials, use of 3D printing, etc. and issues in the 'human (physiology monitoring)' are closely connected with the design of algorithms for the physiological study of people of different age groups, gender, etc. The team decided to focus research on the issues related to product design, human-computer interaction, ergonomics, etc., in the wearable technology design cluster.

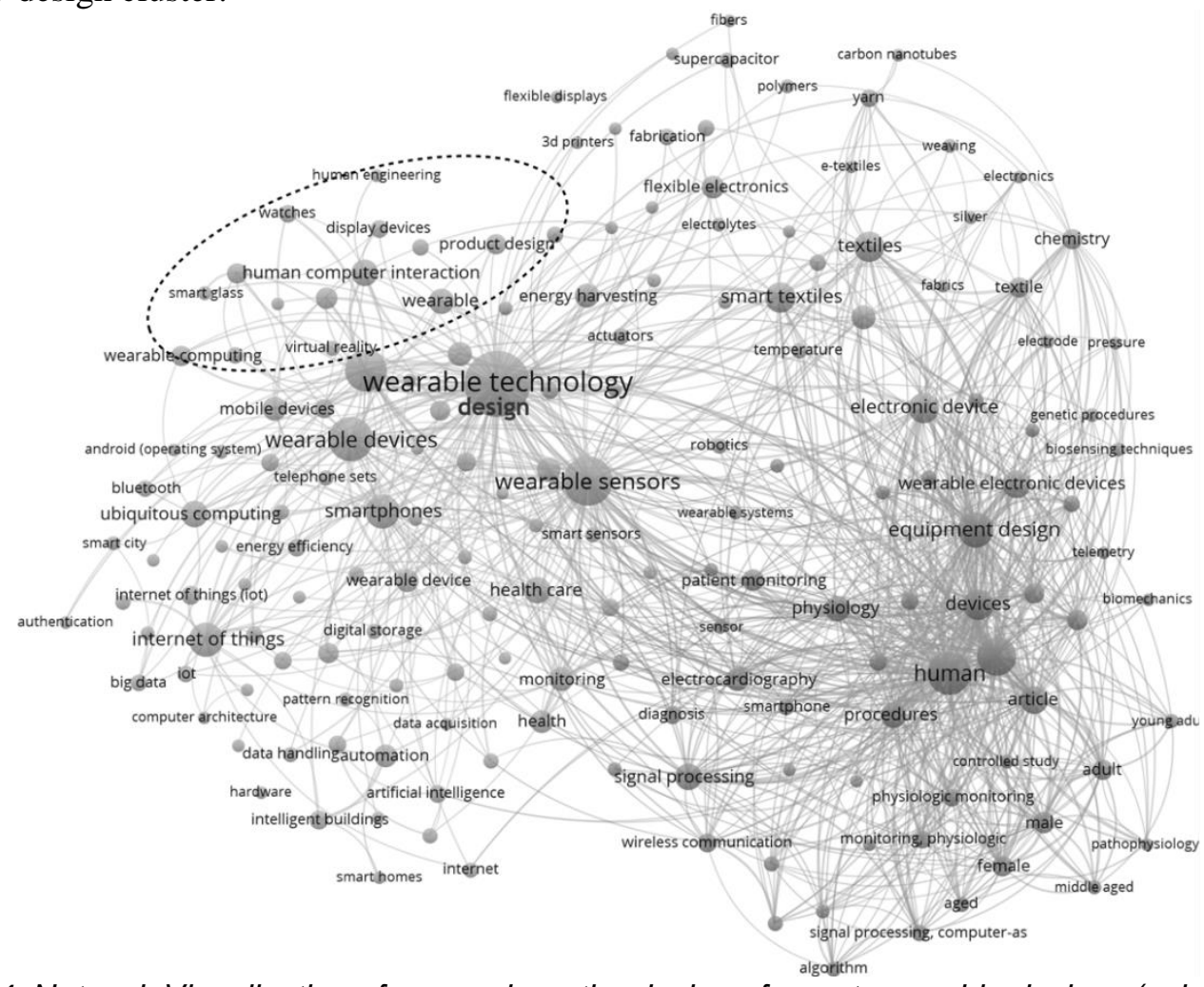

Figure 4. Network Visualization of research on the design of smart wearable devices (using VOS viewer)

Map Trends - Trends reveal a general sense about the most common areas of research opportunities. Recognizing and understanding trends gives a possibility to position the research so that the research findings would indeed be relevant in the near future. The trend search for the current study shows that 
the smart wearable devices market is heavily dominated by wrist-worn devices. Moreover, in the past, shipments of smartwatches were way more than that of wristbands, and the trend will continue to be similar in the future [9]. Thus, the focus object of the current design investigation was determined to be 'smartwatch'.

Articulate Critical Findings - Organizing the critical findings of keywords, identified patterns, and mapped trends in a comprehensive, fact-based format that can be shared with the research team gives a higher-order overview of the interest area of the research as well as forms the rationale for it. It also helps to establish the extent of boundaries of the research landscape. The critical findings of the current case of smart wearable technology design are as follows:

Explosive growth in wearable technology is witnessed in recent years. Smart wearable devices are eventually going to become an integral part of human life [10]. When looked at the current wearable market scenario, the companies in this domain appear to have a strong focus on technology-driven innovation. However, as the market enters the maturation phase, the technology penetration becomes high, and technically similar products co-exist [11]. In such a situation, technology starts losing its potential to become a differentiator, and companies can no longer rely only on technology to gain the upper hand. Therefore, to attain sustainable competitive advantage, companies are desired to set their focus on design with the user at the centre. Emerging technologies like smart wearable devices offer new opportunities for designers to cultivate, enhance, and implement their thoughts and imaginations.

\subsection{Evaluate}

After a full understanding of the intended research area is developed at the end of the 'summarize' stage, the focus moves towards an evaluation through a more context-specific action such as field visit, expert opinion, online/offline survey to gain as many insights as possible through the industry, professional, expert interaction. Here, the primary purpose is to identify practical, "real world" problems that may be understudied or ignored within academic circles and to make research findings more relevant to practice. The evaluation of the currently selected case of smart wearable technology is explained below.

Globally, the smart wearable market devices market is dominated by multinational corporations like Apple, Google, etc. In India, it can be observed that more and more start-ups are venturing into this territory of wearable tech devices. To get recognized in the competitive market, these start-ups hold out against multifaceted challenges. To get further insights on the design challenges encountered by these organizations, expert opinions are gathered through a few informal telephonic interview sessions with practitioners in wearable devices start-ups. The following table shows the particulars of the conducted interviews.

Table 1. The particulars of the conducted interviews

\begin{tabular}{|c|c|c|c|c|}
\hline $\begin{array}{c}\text { Sr } \\
\text { No }\end{array}$ & Company Name & Application Area & Location & $\begin{array}{c}\text { Designation of } \\
\text { interviewee }\end{array}$ \\
\hline 1 & Ducere Technologies & Fitness and Wellness & Telangana, India & Industrial Designer \\
\hline 2 & Ducere Technologies & Fitness and Wellness & Telangana, India & Manager- Product Design \\
\hline 3 & Lazy Co. & $\begin{array}{c}\text { Fashion/Consumer } \\
\text { Electronics }\end{array}$ & Bangalore, India & $\begin{array}{c}\text { CEO \& Industrial } \\
\text { Designer }\end{array}$ \\
\hline 4 & Actofit & $\begin{array}{c}\text { Sports/ Health/ } \\
\text { Fitness and Wellness }\end{array}$ & $\begin{array}{c}\text { Navi Mumbai, } \\
\text { India }\end{array}$ & Founder \& CEO \\
\hline
\end{tabular}

It was quite evident from the interviews that the smart wearable devices industry in India, which is still in infancy, is relying on the designer's abilities and experience in seeking design solutions. However, it takes a lot of practice and professional experience for a designer to gain confidence in decision making in industrial projects [13]. Although traditional design methods have always helped designers deal with practical situations and challenges, what is not paid sufficient attention in these design methods is the complication of contemporary design concerns. Through industrial interaction, it is quite evident that the contemporary design industry (particularly start-ups) needs a novel design method for the ideation stage, which enhances the designer's idea Generation Ability \& Creativity.

At the end of the 'research opportunity identification process' following brief research outline is obtained for the current case: Research gap - The wearable devices start-up industry is in need of a novel design method for the ideation stage which enhances designer's Idea Generation Ability and Creativity; Focus object of design investigation - smartwatch. At this point, the research team can again go back to the 
first stage, i.e., Explore and follow the same loop of explore-categorise-summarise-evaluate in order to further investigate the obtained research gap and gather more profound insights. This process can go on till a refined research gap statement is generated, which satisfies the researcher's interest. Moreover, to cut-short the time consumed in executing the framework, tasks in the multiple stages of the framework can be performed simultaneously, instead of one stage at a time.

\section{DISCUSSION AND CONCLUSION}

This paper proposes a framework for identifying research opportunities describing the key stages and their attributes, explained with a specific case of smart wearable technology design. The proposed pedagogical framework encourages design research students to perform the task of identification of research gap in a much more structured way. Moreover, it is observed during our study that the framework also assists in dealing with the enormous amount of data gathered by organizing it into research relevant areas and thereby reducing the confusion of deciding on which area to focus. This systematic approach in identifying the research gap serves to set the initial point of reference for the research and form the logical basis for it. Thus, the direction of research is evident in the preliminary stages, which helps to refrain from a shift in research focus at later stages.

Interdisciplinary teamwork is becoming the standard in every field as it offers an opportunity to formulate a more comprehensive understanding of an issue that any single discipline may be unable to provide. Designers are increasingly integrated into such interdisciplinary teams because of their creative abilities and skills to humanize technology by thinking beyond functional needs. However, it was observed during the study, the functioning of an interdisciplinary team often turns out to be ineffective due to conflicting working styles and behaviours of the members. In this regard, Belbin's project team model provided insights for building a team with a balance of the team and functional roles. Further, it assisted in assigning appropriate stage leads at different stages of the proposed research opportunity framework. Most importantly, it illustrated how the variations in the team and functional roles could be utilized constructively in an interdisciplinary team.

\section{REFERENCES}

[1] Robinson K. A., Akinyede O., Dutta T., et al. Framework for Determining Research Gaps During Systematic Review: Evaluation [Internet]. Rockville (MD): Agency for Healthcare Research and Quality (US); 2013 Feb. Introduction. Available:

https://www.ncbi.nlm.nih.gov/books/NBK126702/ [Accessed on 2021, 10 January]

[2] Farooq R. (2017). A framework for identifying research gap in social sciences: evidence from the Past.

[3] Michlewski K. Uncovering Design Attitude: Inside the Culture of Designers. Organization Studies, 2008. 29(3): p. 373-392

[4] Rao D. B. Scientific attitude, scientific aptitude, and achievement. 2010: Discovery Publishing House

[5] Belbin R. M. Design Innovation and the TEAM. Design Management Journal (Former Series), 1991. 2(3): p. 38-42

[6] Belbin R. Meredith (1996), Team roles at work, Butterworth Heinemann, Oxford.

[7] The Belbin Team and Bird, V. Belbin, and Project Teams. Available: https://www.belbin.com/resources/blogs/belbin-and-project-teams/ [Accessed on 2021, 25 January], (2018) 20 November.

[8] Tom (2012), "Identifying Gaps in the Literature is a Critical Part of a Review", Available: http://www.literaturereviewof.com/identifying-gaps.html. [Accessed on 2021, 22 January]

[9] Richter F. The Global Wearables Market Is All About the Wrist. Available: https://www.statista.com/chart/3370/wearable-device-forecast/ [Accessed on 2021, 31 January], (2018) 20 September.

[10] Cecchinato M. E., Cox A. L., and Bird J. (2015). Smartwatches: the Good, the Bad and the Ugly? CHI'15 Extended Abstracts, 2133-2138. https://doi.org/10.1145/2702613.2732837

[11] Gonzalez I., Val E., Justel D., and Iriarte I. (2017). A Framework For Product Design Based On Semantic Attribution Process. The Design Journal, 20(sup1), S16-S27. https://doi.org/10.1080/14606925.2017.1352983

[12] Green L. and Bonollo E. (2004). The importance of Design methods to student industrial designers. Global Journal of Engineering Education, 8(2), 175-182. 